\title{
Protection et promotion des droits sociaux des migrants par le Comité européen des droits sociaux
}

Protection and Promotion of Migrant Social Rights by the European Committee of Social Rights

Protección y promoción de los derechos sociales de los migrantes de la parte del Comité Europeo de Derechos Sociales

\section{Marie-Françoise Valette}

\section{OpenEdition}

Journals

Édition électronique

URL : https://journals.openedition.org/remi/7894

DOI : $10.4000 /$ remi.7894

ISSN : $1777-5418$

Traduction(s) :

Protection and Promotion of Migrants' Social Rights by the European Committee of Social Rights URL : https://journals.openedition.org/remi/10420 [en]

Éditeur

Université de Poitiers

Édition imprimée

Date de publication : 1 décembre 2016

Pagination : 337-343

ISBN : 979-10-90426-29-0

ISSN : 0765-0752

Référence électronique

Marie-Françoise Valette, «Protection et promotion des droits sociaux des migrants par le Comité européen des droits sociaux », Revue européenne des migrations internationales [En ligne], vol. 32 - n³ et 4 | 2016, mis en ligne le 01 décembre 2018, consulté le 14 avril 2022. URL : http:// journals.openedition.org/remi/7894; DOI : https://doi.org/10.4000/remi.7894 


\title{
Chronique juridique
}

\section{Protection et promotion des droits sociaux des migrants par le Comité européen des droits sociaux}

\section{Marie-Françoise Valette ${ }^{1}$}

\begin{abstract}
Désormais, les activités de la Cour européenne des droits de l'homme (CEDH) sont suffisamment médiatisées pour que chacun sache que, malgré l'absence de droits spécifiquement liés aux migrations dans la Convention européenne des droits de l'homme, cette juridiction a développé une jurisprudence riche, et contrastée, dans ce domaine. À l'inverse, le Comité européen des droits sociaux (CEDS), chargé du contrôle du respect des obligations étatiques découlant de la Charte sociale ${ }^{2}$ demeure largement dans l'ombre. Cette Charte a pourtant été voulue comme le pendant de la Convention européenne des droits de l'homme, pour les droits sociaux, au sein du Conseil de l'Europe. II faut reconnaître que le CEDS n'est pas une véritable juridiction, mais un organe d'experts, qu'il n'a été créé que près de quarante ans après la $\mathrm{CEDH}^{3}$, que les droits sociaux ont longtemps fait figure de parents pauvres des droits de l'homme et que la Charte sociale n'est pas applicable à tous les étrangers. Cette inégale renommée est surtout accentuée par la possibilité - sous réserve de certaines conditions ${ }^{4}$ - pour tout individu de saisir la CEDH dès lors qu'il se considère victime de violation de droits découlant de la Convention européenne des droits de I'homme. À l'inverse, toujours au sein de l'Europe des quarante-sept, le Comité européen des droits sociaux ne peut faire l'objet que de réclamations collectives émanant d'OING ou de syndicats.
\end{abstract}

Dans le cadre de l'Union européenne, on peut remarquer une situation encore différente. En effet, l'accès de la Cour de justice de I'Union européenne

\footnotetext{
1 Maître de conférences en droit public, Université de Poitiers, MIGRINTER, MSHS, Bât. A5, 5 rue Théodore Lefebvre,TSA 21103, 86073 Poitiers cedex 9 ; marie.francoise. valette@univ-poitiers.fr

2 Pour être rigoureux, il faudrait évoquer les Chartes sociales européennes. Une première version a été signée àTurin en 1961 et une version dite révisée en 1996. Entre les deux, trois protocoles additionnels ont été signés. À l'heure actuelle, certains États ne sont encore liés que par la Charte de 1961.

3 La Cour européenne des droits de I'homme a été créée en 1959. Ce n'est qu'en 1995 qu'un protocole additionnel à la Charte sociale a institué une procédure de réclamations collectives devant le CEDS.

4 L'individu doit être sous la juridiction d'un État membre du Conseil de l'Europe, c'està-dire, le plus souvent, sur son territoire et il doit avoir épuisé les voies de recours internes, c'est-à-dire qu'il doit s'être adressé aux tribunaux de l'Etat concerné.
} 
(CJUE) n'est pas impossible aux individus. Mais pour les questions relatives aux droits des migrants, la CJUE se prononce essentiellement à la suite de questions qui lui sont posées par les juges nationaux - questions préjudicielles - après avoir été eux-mêmes confrontés à une plainte individuelle en lien avec le droit de l'Union.

Jusqu'à la dernière décennie, ces différences ne pouvaient être lues à la lumière d'une quelconque concurrence, notamment dans le champ des droits sociaux des migrants.

Mais le Comité, en interprétant de façon créative, voire audacieuse, la Charte, contribue de plus en plus à promouvoir les droits sociaux des migrants, des réfugiés et des Roms. Il pourrait prétendre à plus de visibilité. Ainsi, I'originalité de son fonctionnement, notamment la place reconnue aux OING, mais aussi les affaires en cours, plus particulièrement celle relative à la prise en charge des mineurs isolés étrangers par la France ${ }^{5}$, et une nouvelle concernant les enfants Roms $^{6}$, donnent à cette quasi-juridiction un intérêt croissant dans le domaine des migrations, à défaut d'une large audience. En même temps, la Cour européenne étend de plus en plus clairement sa compétence aux droits économiques et sociaux et I'Union européenne, avec le traité de Lisbonne, a donné une valeur contraignante à sa Charte des droits fondamentaux qui conjuguent droits économiques et sociaux et droits civils et politiques.

Paradoxalement, ces évolutions risquent d'enfoncer plus encore dans l'ombre le CEDS (Akandji-Kombé, 2014) alors même qu'elles devraient être perçues comme autant de raisons de faire connaître ses activités au-delà d'un petit cercle de spécialistes.

\section{L'affirmation des droits sociaux}

Après que leur juridicité a été remise en cause, les droits sociaux apparaissent désormais indiscutablement justiciables (Roman, 2012) et de plus en plus souvent indissociables des droits civils et politiques, alors que leurs liens mutuels ont été objets de controverses.

\section{Les droits sociaux, parents pauvres des droits de l'homme}

Longtemps tout caractère obligatoire a été dénié aux droits économiques et sociaux, notamment en raison d'une confusion entre absence d'obligation de résultat et absence d'obligation. Leur dimension politique et coûteuse apparaissait comme incompatible avec tout véritable engagement. Or, le CEDS, mais aussi à I'ONU, le Comité des droits économiques, sociaux et culturels, ont su mettre en avant les exigences de la réalisation progressive des droits et l'importance des obligations positives incombant aux États indépendamment des catégories traditionnelles. C'est ainsi que le CEDS a rappelé en octobre 2015 ce qu'il avait déjà eu l'occasion d'énoncer en 2003 : " Lorsque la réalisation d'un droit en question est exceptionnellement complexe et particulièrement onéreuse, les États parties doivent s'efforcer d'atteindre les objectifs de la Charte à une

5 Réclamation $n^{\circ} 114 / 2015$ présentée par EUROCEF le 27 février 2015 contre la France.

6 Réclamation $n^{\circ} 119 / 2015$ présenté par FERV le 19 octobre 2015 contre la France. 
échéance raisonnable, au prix de progrès mesurable, en utilisant au mieux les ressources qu'ils peuvent mobiliser $" 7$. Cependant, trop souvent les distinctions entre droits dits de la première génération, de la deuxième et de la troisième, ou entre les droits libertés (droits de) et les droits créances (droits à) sont encore mises en avant. Or aujourd'hui, même les néolibéraux reconnaissent que tous les droits sont susceptibles d'avoir un " coût " (Lacroix et Pranchère, 2016).

\section{L'indivisibilité des droits de I'homme}

La Déclaration universelle des droits de l'homme n'opérait pas de distinction entre les droits civils et politiques et les droits économiques, sociaux et culturels. Par la suite, à l'échelle universelle, l'adoption, en 1966, de deux Pactes portant respectivement sur les uns et sur les autres, a contribué à ancrer cette séparation. Au niveau régional, les États membres du Conseil de l'Europe ont adopté dès l'origine un traité restreint. Aucun droit social ou économique ne figure dans la Convention européenne des droits de I'homme.

L'indivisibilité et l'interdépendance de ces différents droits de l'homme sont reconnues aujourd'hui, tant par la Cour européenne, que par le CEDS.

\section{L'interprétation évolutive du champ d'application personnel de la Charte sociale européenne}

Bien que depuis la fin de la Seconde Guerre mondiale, la protection internationale des droits de l'homme ait acquis une dimension objective, le champ d'application personnel de la Charte sociale européenne demeure limité. Contrairement aux autres traités régionaux ou universels, ses dispositions ne sont a priori pas applicables à toute personne sous le contrôle d'un État partie.

\section{Les migrants inclus dans la définition du champ d'application}

Trois catégories de non-ressortissants de l'État de résidence sont très explicitement prises en compte dans les deux Chartes. II s'agit des travailleurs migrants, des réfugiés et des apatrides:

- Une distinction est opérée au sein des travailleurs migrants entre ceux qui ont la nationalité d'un autre État membre du Conseil de l'Europe et partie à la Charte, et les autres travailleurs étrangers. La Charte comprend un article spécifiquement consacré aux travailleurs migrants et à leur famille qui semble inclure l'ensemble des travailleurs étrangers, tout en restant un peu confus. De toute façon, des différences de traitement peuvent être conformes à ce traité. Autrement dit, tous les droits garantis par la Charte ne bénéficient pas automatiquement aux travailleurs étrangers.

- Les réfugiés sont évoqués à l'annexe des deux versions de la Charte, tout comme le sont les apatrides.

7 Observation interprétative sur Les droits des réfugiés au regard de la Charte sociale européenne, 21, le 5 octobre 2015, [en ligne] consulté le 02/08/2016. URL : http://hudoc. esc.coe.int/fre/?i=2015_163_10/FR 
Le CEDS, alarmé, comme l'ensemble des organes du Conseil de l'Europe ${ }^{8}$, par la situation des réfugiés à partir de l'été 2015, a tenu à préciser l'acception qui devait être retenue pour cette catégorie de migrants, ainsi que leurs droits. $C^{\prime}$ est ainsi qu'il a adopté une relativement longue observation interprétative sur ces questions, afin de guider les États, dans la mise en œuvre des droits garantis par la Charte. II a notamment précisé que les réfugiés devaient être protégés par la Charte dès lors que leur situation correspond à celle qui est définie par la Convention de 1951, et ce indépendamment " de la reconnaissance administrative du statut de réfugié par un État, laquelle de fait par l'octroi de l'asile ".

\section{L'extension de certains droits aux migrants en situation irrégulière}

La conception objective de la protection internationale des droits de I'homme ne permet d'exclure aucun individu de ce bénéfice. Cependant, seule la Convention sur les droits des travailleurs migrants et de leur famille, adoptée dans le cadre de I'ONU en 1996, mentionne explicitement les droits des migrants en situation irrégulière, au prix de l'absence de participation de tous les États d'immigration du Nord.

Au nom de la dignité humaine, le CEDS a donné une interprétation très extensive du champ d'application personnel de la Charte sociale, incluant clairement les migrants en situation irrégulière. Le recours à l'interprétation téléologique I'a conduit, d'abord de façon hésitante (Daugareilh, 2005), puis avec plus de fermeté (Nivard, 2014), à élargir les obligations étatiques, pour un certain nombre de droits considérés comme essentiels. C'est ainsi qu'un pas, esquissé en 2004, a bien été franchi en juillet 2014, à travers deux décisions concernant les Pays-Bas. Dans les deux cas étaient en cause des restrictions d'accès à des aides d'urgence. La nécessité de couvrir des besoins essentiels avait même conduit le CEDS à adresser des " mesures immédiates " au gouvernement néerlandais, ce qui reste tout à fait exceptionnel.

Ces extensions du champ d'application de la Charte sociale sont d'autant plus remarquables que non seulement elles ne découlent pas du tout des textes dont le CEDS est supposé veiller au respect, mais qu'en plus aucun des États parties, sollicités en 2011 pour étendre le bénéfice de droits sociaux à toute personne sous leur juridiction, n'a, jusqu'à ce jour, répondu favorablement (Nivard, 2014).

\section{Les mécanismes de contrôle exercés par le Comité européen des droits sociaux}

À l'origine, la Charte de 1961 ne prévoit qu'une sorte de contrôle, le contrôle sur rapports, par un Comité d'experts, sous l'autorité d'un organe composé de représentants des États. Mais, en 1995, un système de réclamations collectives a été introduit, afin de donner un nouvel élan à la Charte sociale européenne (Sudre, 1996). Dans un cas comme dans l'autre, les conclusions et décisions du CEDS doivent être entérinées par le Comité des ministres du Conseil de

8 Une préoccupation notamment traduite par la nomination, en janvier 2016, de Tomáš Boček comme Représentant Spécial du Secrétaire Général du Conseil de I'Europe pour les migrations et les réfugiés. 
I'Europe, composé de représentants des quarante-sept États membres. Ces deux mécanismes sont inspirés de ce qui existe à l'Organisation internationale du travail (OIT).

\section{Les contrôles sur rapports}

Chaque État partie a l'obligation de remettre une fois par an au Comité un document par lequel il expose les conditions d'application des dispositions qu'il a acceptées. II peut notamment expliquer les difficultés qu'il a rencontrées et la façon dont il a pris en compte les remarques faites lors de l'examen d'un précédent rapport.

Ce type de contrôle est fréquent en droit international. C'est ainsi que chacun des traités relatifs à la protection des droits de l'homme adopté dans le cadre des Nations unies prévoit un mécanisme comparable ${ }^{9}$. II en va de même à I'Organisation internationale du travail (OIT) où il existe depuis 1926 ! Or, cette organisation œuvre dans des domaines proches de ceux du CEDS et est étroitement associée à ses travaux.

Depuis 2006, afin d'alléger la charge de travail, tant des États que du CEDS, chaque disposition n'est examinée que tous les quatre ans. Dans cette optique, les droits garantis ont été regroupés en quatre thématiques. L'un de ces ensembles est composé des obligations liées aux enfants, à la famille et aux migrants. C'est celui qui était retenu pour l'exercice 2015. Pour chaque thématique, le CEDS détermine des questions. À titre d'exemple, étaient en cause les tests de langue et d'intégration et les conditions de logement imposés dans un objectif de regroupement familial, mais également les possibilités d'accès au logement ou les mesures prises en faveur des populations vulnérables telles que les Roms.

\section{Les réclamations collectives}

Le droit de réclamation est réservé aux syndicats et aux organisations internationales non gouvernementales dotées du statut consultatif auprès du Conseil de l'Europe, inscrites sur une liste spéciale et reconnues compétentes dans le domaine sur lequel porte la réclamation ${ }^{10}$. Elles sont actuellement soixantedouze. Ce droit ne peut être exercé que contre les États l'ayant reconnu, soit quinze États à la fin du mois de juillet 2016. Les réclamations qui ont été jusqu'au terme de la procédure sont le fait d'OING et de syndicats assez diversifiés. Cependant, le Centre européen des droits des Roms se distingue avec déjà huit réclamations menées à leur terme. Cette OING forme ses membres sur les réclamations collectives et leur explique en particulier l'intérêt de connaître les rapports établis par le CEDS sur chaque État afin d'argumenter de façon efficace

9 Notamment l'analyse du rapport de la France par le Comité des droits de l'enfant des Nations unies, Observations finales concernant le cinquième rapport périodique de la France, janvier 2016, [en ligne] consulté le 02/08/2016. URL : https://documents-dds-ny. un.org/doc/UNDOC/GEN/G16/033/67/PDF/G1603367.pdf

10 Sous certaines conditions, une ONG purement nationale peut également faire une réclamation collective. 
les plaintes ${ }^{11}$. Par ailleurs, elle propose un bilan des réclamations dans lequel elle relève celles qui ont abouti à une modification des législations en cause. Elle suggère de saisir les médias aux fins de pressions sur les États récalcitrants.

L'intérêt de ce type de plainte est qu'il conduit le Comité à connaître non pas des situations individuelles, mais des situations communes à des groupes de personnes. Il s'agit pour une organisation de la société civile de dénoncer une pratique ou une législation étatique non conforme aux dispositions de la Charte qui lient l'État objet de la plainte. Le choix de ce mécanisme, et non d'un système juridictionnel comparable à celui de la $\mathrm{CEDH}$, est fréquemment déploré et considéré comme une occasion manquée de donner aux droits sociaux la même chance d'effectivité qu'à ceux garantis par la Convention européenne des droits de I'homme (Sudre, 1996). Toutefois il présente certains avantages. $C^{\prime}$ est ainsi que, contrairement aux recours devant la $\mathrm{CEDH}$, les auteurs de la réclamation ne sont pas directement des victimes, mais sont en quelque sorte des lanceurs d'alerte. Ils utilisent la " force du droit " (Israël, 2009) et permettent l'établissement d'un lien étroit entre revendications militantes et système quasi juridictionnel.

Au terme d'une procédure assez longue permettant à l'État visé de répondre de façon détaillée à la plainte, le CEDS adopte et rend publique une décision dite sur le bien-fondé. Celle-ci est officiellement transmise aux parties, mais également au Comité des ministres du Conseil de l'Europe qui peut adopter une recommandation à l'encontre de l'État qui ne manifesterait pas la volonté de suivre le CEDS.

Ces mécanismes s'apparentent à un " monitoring " des obligations découlant de la Charte sociale. S'ils ne se concluent ni dans le cas des rapports, ni dans le cas des réclamations collectives par des décisions exécutoires, ils doivent néanmoins être suivis par les autorités nationales.

\section{Références bibliographiques}

Akandji-Kombé Jean-François (2014) Droit constitutionnel, droit international et droit européen des droits de l'homme : concurrence, confusion, complémentarité ?, Droit social, 4, pp. 301-307.

Boissard Béatrice (2010) La contribution du comité européen des droits sociaux à l'effectivité des droits sociaux, Revue du droit public et de la science politique en France et à l'étranger, 4, pp. 1053-1111.

Daugareilh Isabelle (2005) L'audace retenue du Comité européen des droits sociaux. À propos de la décision FIDH c/ France réclamation n¹4/2003, Revue de droit sanitaire et social, pp. 555-564.

Israël Liora (2009) L'arme du droit, Paris, Les Presses de Sciences Po, 142 p.

11 Compte-rendu de la formation destinée aux personnes impliquées dans la défense du droit des Roms et voyageurs, 19-20 février 2009, [en ligne] consulté le 04/08/2016. URL : http://romeurope.org/lMG/pdf/Les_20reclamations_20collectives_20au_20Conseil_20de _20l_Europe.pdf 
Lacroix Justine et Pranchère Jean-Yves (2016) Le procès des droits de I'homme. Généalogie du scepticisme démocratique, Paris, Seuil, 352 p.

Marguénaud Jean-Pierre et Mouly Jean (2011) Le Comité européen des droits sociaux, un laboratoire d'idées sociales méconnu, Revue du droit public, 3, pp. 685-716.

Nivard Carole (2014) Précisions sur les droits de la Charte sociale européenne bénéficiant aux étrangers en situation irrégulière, La Revue des droits de I'homme, consulté le 27/01/2016 [en ligne]. URL : https://revdh.revues.org/982

Roman Diane (2012) La justiciabilité des droits sociaux ou les enjeux de l'édification d'un État social, La revue des droits de l'homme, consulté le 29/07/2016 [en ligne]. URL : revdh.revues.org/635

Sudre Frédéric (1996) Le protocole additionnel à la Charte sociale européenne prévoyant un système de réclamations collectives, Revue Générale de Droit International Public, 100 (3), pp. 715-739. 